\title{
Identifying Emergency Department Care in the Year Prior to Suicide Death
}

\author{
Dylan Delisle 1, 2, Anna E. Waller1, 2, Katherine Wolff 1, 2, Katherine J. Harmon 1, 2, 3, Amy \\ Ising ${ }^{1,2}$ \\ ${ }^{1}$ Carolina Center for Health Informatics, Dept. of Emergency Medicine, UNC-Chapel Hill, Chapel Hill, North Carolina, United States, ${ }^{2}$ Carolina Center \\ for Health Informatics, Chapel Hill, North Carolina, United States, ${ }^{3}$ Highway Safety Research Center, Chapel Hill, North Carolina, United States
}

Objective

To identify potential emergency department (ED) visits prior to suicide deaths in North Carolina (NC) and describe pre-suicide care-seeking in EDs.

\section{Introduction}

Suicide is a leading cause of mortality in the United States, causing about 45,000 deaths annually [1]. Research suggests that universal screening in health care settings may be beneficial for prevention, but few studies have combined detailed suicide circumstances with ED encounter data to better understand care-seeking behavior prior to death.

\section{Methods}

This project used data from the NC Violent Death Reporting System (NC-VDRS), a repository of all violent deaths in North Carolina, and the NC Disease Event Tracking and Epidemiologic Collection Tool (NC DETECT), which includes all visits to 24/7, acute-care, civilian, hospital-affiliated EDs in NC. We identified all suicide deaths recorded in the NC-VDRS between 1/1/2014 9/30/2015, and all NC DETECT ED visits between 1/1/2013 - 9/30/2015. Descriptive analyses were conducted for each data source, separately. For all NC-VDRS suicides, we identified ED visits likely made by the same individual within the 48 hours prior to death. We identified these ED visits based on the variables arrival date, sex, date of birth (DOB), county of residence, and a chief complaint consistent with self-harm/suicide; we refer to these as suicide-related ED visits. For the subset of NC-VDRS suicides with a suicide-related ED visit, made within 48 hours of death, we identified all ED visits associated with the decedent made to the same facility or healthcare system in the year prior to death. We then categorized the pre-suicide ED visits according to the primary reason healthcare was sought by the patient (e.g. mental health problem, substance abuse/overdose, pain, etc.).

\section{Results}

From 1/1/2014-9/30/2015, there were 2,953 suicide deaths captured in NC-VDRS data; 2,435 (82\%) of these included DOB. Between 1/1/2013 - 9/30/2015, there were 13,463,345 ED visits captured by NC DETECT; 12,884,596 (96\%) included DOB. For 961 suicides (32.5\%), no ED visit was found with the same DOB, sex and county of residence. For the remaining 1,474 suicides, at least one ED visit was found for a patient with the same DOB, sex and county of residence and occurring on or before the date of death. For 406 suicides, a suicide-related ED visit was identified; 122 of these patients had at least one additional ED visit in the year prior to death. A total of $516 \mathrm{ED}$ visits were identified for these 122 suicides, including the suicide-related ED visit, with an average of 3.2 (range: $1-25$ ) visits.

Gender, race and mechanism of death were similar between those with an ED visit prior to death and the total suicide population (Table 1). The chief complaint and diagnosis codes for the 394 prior ED visits varied. The most frequent diagnoses/chief complaints were long term medication use (35.3\%), hypertension (29.9\%), pain (20.8\%), and diabetes mellitus (18.8\%). Depression was coded for 62 visits (15.7\%); only 16 visits (4.1\%) received a code for suicide ideation. Of the 122 patients with both a suicide-related ED visit and at least one prior ED visit, NC-VDRS data indicated that 28 individuals (23.0\%) were perceived as depressed at the time of death and 46 individuals (37.7\%) had a history of suicidal thoughts. At the time of death, 32 individuals (26.4\%) had alcohol in their system. In the NC-VDRS data, mental health problems were documented for a higher percentage of males with a suiciderelated ED visit than the total male suicide population (56.9\% versus $47.0 \%)$, and a higher percentage were in treatment for mental health issues at the time of death $(50.0 \%$ versus $40.0 \%)$. Conversely, females with a suicide-related ED visit had a lower percentage of documented mental health problems and treatment $(66.7 \%$ versus $71.0 \%$, and $55.6 \%$ versus $64.0 \%$, respectively) than the total female suicide population in NC-VDRS. 


\section{Conclusions}

For nearly a third of NC-VDRS suicides, no indication of any ED visit by a patient with the same DOB, sex, and county of residence was found. While it is likely we were unable to identify all ED visits prior to suicide, the findings from this pilot study suggest many suicide victims did not seek NC ED care in the year prior to death. Overall, a suicide-related ED visit was found for only $13.7 \%$ of NC suicides in the study period, indicating that most people who self- inflict fatal injury do not make it to an ED for care prior to death. ED visits in the year prior to death by suicide indicated a variety of diagnoses, but rarely depression or suicidality; this suggests that universal screening at ED visits would have been necessary to identify any suicide risk present. Limitations of this study include that we were unable to directly link suicide deaths and ED visits using patient identifiers. Additionally, we relied solely on secondary data used for public health surveillance and, therefore, had no access to medical record information that may have documented depression or suicidal ideation that was not coded as such. Findings from this pilot study can inform future work to identify ED visits prior to suicide.

\section{Acknowledgement}

NC DETECT is a statewide public health syndromic surveillance system, funded by the NC Division of Public Health (NC DPH) Federal Public Health Emergency Preparedness Grant and managed through collaboration between NC DPH and UNC-CH Department of Emergency Medicine's Carolina Center for Health Informatics. The NC DETECT Data Oversight Committee does not take responsibility for the scientific validity or accuracy of methodology, results, statistical analyses, or conclusions presented.

The North Carolina Violent Death Reporting System (NC-VDRS) is a public health, population-based surveillance system that contains detailed information on deaths that result from violence. The NC-VDRS is an incident-based, relational database that combines information from multiple sources, such as death certificates, medical examiner reports, and incident reports from law enforcement agencies. It is operated by the North Carolina Division of Public Health's Injury and Violence Prevention Branch to provide injury and violence prevention specialists and policy-makers with timely information on the victims, suspects, relationships, circumstances, and weapons that are associated with every incident of violence that results in a fatality in North Carolina.

\section{References}

1. National Center for Injury Prevention and Control. Suicide Rising Across the US. Vital Signs, June 2018; Atlanta, GA: Centers for Disease Control and Prevention 2018. https://www.cdc.gov/vitalsigns/suicide/. Accessed Sept 25, 2018.

Table 1. Demographic Information for Total Suicide Population, Suicides with ED Visit Within 48 Hours of Death, and Suicides with ED Visits in Year Prior to Death, North Carolina, 1/1/2014-9/30/2015

\begin{tabular}{|c|c|c|c|c|}
\hline & & $\begin{array}{c}\text { Total Suicide } \\
\text { Population }\end{array}$ & $\begin{array}{l}\text { Suicides with ED visit within } 48 \text { hours of } \\
\text { death (a) }\end{array}$ & $\begin{array}{l}\text { Suicides with additional ED visits in year } \\
\text { prior to death (b) }\end{array}$ \\
\hline Population & $\mathrm{N}$ & 2953 & 406 & 122 \\
\hline Age & Mean & 47.8 & 47.5 & 50.9 \\
\hline \multirow[t]{2}{*}{$\operatorname{Sex}(\%)$} & Male & 74.3 & 74.8 & 72.1 \\
\hline & Female & 25.7 & 25.2 & 27.9 \\
\hline \multirow[t]{2}{*}{ Race $(\%)$} & White & 86.4 & 84.2 & 87.7 \\
\hline & Black & 8.9 & 7.9 & 4.9 \\
\hline \multirow{3}{*}{$\begin{array}{c}\text { Mechanism } \\
(\%)\end{array}$} & Firearm & 56.2 & 61.2 & 52.5 \\
\hline & Suffocation & 20.7 & 19.3 & 18.9 \\
\hline & Poisoning & 18.3 & 15.8 & 27.1 \\
\hline
\end{tabular}

(a) This column represents the subset of the Total Suicide Population for whom an ED visit could be identified in the 48 hours prior to death. (b) This column represents the subset of the Suicides with ED visit within 48 hours of death for whom additional ED visits in the year prior to death could be identified. 